\title{
Geometric adeles and the Riemann-Roch theorem for 1-cycles on surfaces
}

\author{
Ivan Fesenko
}

\begin{abstract}
The classical Riemann-Roch theorem for projective irreducible curves over perfect fields can be elegantly proved using adeles and their topological self-duality. This was known already to E. Artin and K. Iwasawa and can be viewed as a relation between adelic geometry and algebraic geometry in dimension one. In this paper we study geometric two-dimensional adelic objects, endowed with appropriate higher topology, on algebraic proper smooth irreducible surfaces over perfect fields. We establish several new results about adelic objects and prove topological self-duality of the geometric adeles and the discreteness of the function field. We apply this to give a direct proof of finite dimension of adelic cohomology groups. Using an adelic Euler characteristic we establish an additive adelic form of the intersection pairing on the surfaces. We derive a direct and relatively short proof of the adelic Riemann-Roch theorem. Combining with the relation between adelic and Zariski cohomology groups, this also implies the Riemann-Roch theorem for surfaces.
\end{abstract}

The adelic point of view in one-dimensional algebraic and arithmetic geometry not only leads to proofs of key properties but often explains underlying reasons for these properties to hold. In particular, adeles for a proper curve over a field can be used to prove and explain several geometrical properties of the curve. Topological self-duality of adeles and the structure of their subquotients essentially imply the Riemann-Roch theorem and what was later called Serre's duality for projective smooth irreducible curves. A nice presentation can be found in Artin's lectures for function fields over finite fields [A, Ch.XIV]. This was extended in [Iw2], [Iw3] to projective smooth irreducible curves over any field, and in $[\mathrm{G}]$ to projective irreducible curves over any field. One can also use appropriate portions of the zeta integral theories of [Iw1], [T, 4.2], [W], where one works not only with the adelic objects but with non-linear functions on them, to get longer proofs of the RiemannRoch theorem which use translation invariant measure and integration, Fourier transform and elements of harmonic analysis on appropriate spaces of functions on adeles and their subquotients. The proof in the book [W, Ch.VI] is essentially measure and integration free.

Higher-dimensional geometric rational adeles for algebraic varieties over fields were described in [P1] and full adeles were outlined in [B1], [P2]. Their further theory and applications have already led to many interesting development. More recently, it was discovered that there are other adelic structures in higher dimensions [F3]. In particular, on schemes of dimension two there are two different adelic structures. One structure is of more geometric nature, closely related to 1-cocycles. While the geometric adelic structure is good for an adelic proof of the Riemann-Roch theorem, the other structure is of more analytic nature, [F3, Ch.2], [F4, Ch.1], closely related to 0-cycles, and it is here that one has a generalisation of translation invariant measure, integration and harmonic analysis, and a generalization of the Iwasawa-Tate theory to two-dimensional adelic zeta integrals over 
the analytic adeles, with many applications to the zeta functions of arithmetic schemes, [F3], [F4]. Unlike the classical case of dimension one, there seems to be no single adelic structure which can cover both the 1-cycles and 0-cycles aspects on surfaces. Geometric and analytic aspects on surfaces are quite different worlds, unlike the case of curves, and often interactions between these aspects, such as the BSD conjecture, can be viewed as a nontrivial relation between the two adelic structures.

The paper [P1] posed the problem to find an adelic proof of the Riemann-Roch theorem for divisors on projective smooth irreducible surfaces. A sketch of line of thought to solve this problem was recently announced in [OP2] without proofs of several key statements. In addition to the older foundational papers [P1], [B1], [P2], the incomplete argument in [OP2] essentially relies on lengthy texts [OP1].

Artin's adelic proof of the Riemann-Roch theorem in dimension one is very short, see section $\mathbf{0}$ for a compact presentation. For further developments of adelic geometry, as well as for applications of the two-dimensional zeta integral to the study of fundamental properties of zeta functions [F4], [F5], it would be good to have a direct and relatively short proof of the Riemann-Roch theorem in dimension two. This paper tries to address this need. The method of this paper can be viewed as an extension of both the one-dimensional topological adelic method and the foundations of the two-dimensional theory in [P1] and [B1]. In comparison to [P1] we use more intensive topological considerations which are quite powerful when combined with arithmetic and geometric issues. They give short proofs of key results, without the use of [OP1-OP2]. It is essentially topological self-duality of the full geometric adeles which underlies the Riemann-Roch theorem and Serre duality. We hope that this paper may appeal to a wider class of readers interested in adelic geometry and its applications.

Some of results in this work are extensions of those in [P1] for rational geometric adeles to the full geometric adeles. This extension can sometimes be nontrivial and in those cases we include proofs, discussions and corrections. Novel issues in this paper include several new results about the topology of the geometric adeles and their subobjects, the study of topological self-duality of the geometric adeles and its applications, a proof of the discreteness of the function field in the geometric adeles, a direct proof of finite dimension of adelic cohomology groups without using Zariski cohomology groups, a definition of adelic Euler characteristic independently of Zariski cohomology Euler characteristic, an additive adelic form of the intersection pairing on surfaces, a proof of the equality of the adelic intersection pairing and the usual intersection pairing, a direct and short proof of the adelic Riemann-Roch theorem and finally an adelic proof of the Riemann-Roch theorem for proper smooth irreducible surfaces over arbitrary perfect fields.

Basic prerequisites about two-dimensional local fields and the geometric adeles for this paper are standard, they are referred to in appropriate places of this paper. For main results about higher local fields relevant for this work, see papers in the volume [FK] and the recent compact survey [M3] which also includes a short presentation of algebraic aspects of the geometric adeles.

After giving the main definitions in section 1, we study a number of useful properties of the geometric adeles in section $\mathbf{2}$ and $\mathbf{3}$. Then we show how topological self-duality of the geometric adeles and the moving lemma, i.e. an approximation property, imply the 
new adelic description of the intersection pairing. The latter easily gives, as corollary, a short proof of the adelic Riemann-Roch theorem in dimension two. By applying only at the end the known relation between Zariski cohomologies and adelic complex cohomology from [P1], [B1], [H] we immediately see that the adelic Riemann-Roch theorem implies the classical Riemann-Roch theorem on surfaces.

The adelic description of the intersection index in this paper involves the additive group of the geometric adeles only, and not $K$-groups of the adelic objects which are in use in [P2]. This opens a number of further opportunities to use the results of this paper.

One of advantages of adelic methods is their uniform applicability in different characteristics. An extension of this work to the case of arithmetic surfaces is expected to provide an adelic interpretation of the Arakelov intersection pairing and another proof of the Faltings-Riemann-Roch theorem.

0. Here is a sketch of how the classical one-dimensional proof can be conducted. The exposition in this section is essentially based on [A], [Iw2], [G].

Let $\mathrm{k}$ be the function field of a smooth proper irreducible curve $C$ over a perfect field $\mathbb{F}$. We work with $\mathbb{F}$-linear topologies and $\mathbb{F}$-linear compactness, the notions from the classical text [Le,II, $\S 6]$. The field $\mathbb{F}$ is endowed with the discrete topology, the local fields associated to closed points of $C$ are endowed with the topology of local fields and their rings of integers are linearly compact. Adeles are endowed with the restricted product topology. The adelic $\mathbb{F}$-algebra $\mathbb{A}_{k}$ contains the $\mathbb{F}$-algebra $\mathbb{A}_{k}(0)$ which is the product of the completions of the local rings of $C$ at its closed points. For a divisor $d$ on the curve we have an adelic complex

$$
\mathcal{A}_{\mathrm{k}}(d): \quad \mathrm{k} \oplus \mathbb{A}_{\mathrm{k}}(d) \longrightarrow \mathbb{A}_{\mathrm{k}}, \quad(a, b) \mapsto a-b
$$

where $\mathbb{A}_{\mathrm{k}}(d)=\alpha_{d} \mathbb{A}_{\mathrm{k}}(0)$ for any $\alpha_{d} \in \mathbb{A}_{k}^{\times}$such that $v\left(\alpha_{d}\right)=-v(d), v$ runs through all discrete valuations on $\mathrm{k}$, i.e. all closed points of the curve $C$, and $d=\sum v(d) d_{v}, d_{v}$ is the class of the valuation/closed point in the divisor group.

This complex is quasi-isomorphic to the complexes

$$
\mathbb{A}_{\mathrm{k}}(d) \longrightarrow \mathbb{A}_{\mathrm{k}} / \mathrm{k}, \quad \mathrm{k} \longrightarrow \mathbb{A}_{\mathrm{k}} / \mathbb{A}_{\mathrm{k}}(d) .
$$

We have $H^{0}\left(\mathcal{A}_{\mathrm{k}}(d)\right)=\mathrm{k} \cap \mathbb{A}_{\mathrm{k}}(d), H^{1}\left(\mathcal{A}_{\mathrm{k}}(d)\right)=\mathbb{A}_{\mathrm{k}} /\left(\mathrm{k}+\mathbb{A}_{\mathrm{k}}(d)\right)$.

Define a map $\left(\alpha_{v}\right) \mapsto \sum_{v} \operatorname{Tr}_{k(v) / \mathbb{F}} \operatorname{res}_{v}\left(\alpha_{v} \omega\right)$ for a differential form $\omega \in \Omega_{\mathrm{k} / \mathbb{F}}^{1}$, where $k(v)$ is the residue field of the local ring at $v$. Composing with the multiplication $\mathbb{A}_{\mathrm{k}} \times \mathbb{A}_{\mathrm{k}} \longrightarrow \mathbb{A}_{\mathrm{k}}$ we get the differential pairing

$$
\mathbb{A}_{\mathrm{k}} \times \mathbb{A}_{\mathrm{k}} \longrightarrow \mathbb{F}, \quad(\alpha, \beta) \mapsto \sum_{v} \operatorname{Tr}_{k(v) / \mathbb{F}} \operatorname{res}_{v}\left(\alpha_{v} \beta_{v} \omega\right)
$$

Using self-duality of the $\mathbb{F}$-space of a local field it is easy to prove that the space $\mathbb{A}_{k}$ is (non-canonically) self-dual: the $\mathbb{F}$-linear space of continuous linear maps from the $\mathbb{F}$-space $\mathbb{A}_{k}$ to $\mathbb{F}$ is non-canonically algebraically and topologically isomorphic to the $\mathbb{F}$-space $\mathbb{A}_{k}$. 
If $C$ is a projective line over $\mathbb{F}$, so $\mathrm{k}=\mathbb{F}(t)$, it is easy to see that $\mathbb{A}_{\mathrm{k}}$ is the direct sum of $\mathrm{k}$ and a linearly compact space $R$ where $R$ is the product of the rings of integers of the local fields for all discrete valuations different from that one which has $t^{-1}$ as a prime element, for the latter its component of $R$ is the maximal ideal of the corresponding local field. This property extends to the general case using the trace map from $\mathrm{k}$ down to $\mathbb{F}(t)$. Hence the space $\mathbb{A}_{\mathrm{k}} / \mathrm{k}$ is linearly compact. The complement $\mathrm{k}^{\perp}$ of $\mathrm{k}$ with respect to the differential pairing is a $\mathrm{k}$-space which contains $\mathrm{k} ; \mathrm{k}^{\perp} / \mathrm{k}$ is a closed subspace of $\mathbb{A}_{k} / k$, hence linearly compact, on the other hand, $k^{\perp}$ is the space of continuous linear maps from the linearly compact $\mathbb{A}_{\mathrm{k}} / \mathrm{k}$ to $\mathbb{F}$, hence it is discrete, then $\mathrm{k}^{\perp} / \mathrm{k}$ is discrete and linearly compact, hence of finite $\mathbb{F}$-dimension, since $\mathrm{k}$ is of infinite $\mathbb{F}$-dimension, we deduce $\mathrm{k}^{\perp}=\mathrm{k}$ (the argument in this sentence is from [T, Th.4.1.4]).

For an $\mathbb{F}$-subspace $H$ of the adeles denote $H^{\perp}=\left\{\beta \in \mathbb{A}_{\mathrm{k}}:(H, \beta)=0\right\}$. The complement $\mathbb{A}_{k}(0)^{\perp}$ of $\mathbb{A}_{k}(0)$ with respect to the pairing is $\mathbb{A}_{k}(\mathfrak{c}), \mathfrak{c}$ is the divisor of $\omega$ and is called the canonical divisor. We get $\mathbb{A}_{k}(d)^{\perp}=\mathbb{A}_{k}(\mathfrak{c}-d)$, hence the space of continuous linear maps from $H^{0}\left(\mathcal{A}_{\mathrm{k}}(d)\right)$ to $\mathbb{F}$ is isomorphic to $\mathbb{A}_{\mathrm{k}} / H^{0}\left(\mathcal{A}_{\mathrm{k}}(d)\right)^{\perp}$, i.e. to $H^{1}\left(\mathcal{A}_{\mathrm{k}}(\mathfrak{c}-d)\right)$. The space $\mathbb{A}_{k}(0)$ and hence $\mathbb{A}_{k}(d)$ are linearly compact, and their intersection with $\mathrm{k}$ is discrete, which implies that $H^{0}\left(\mathcal{A}_{\mathrm{k}}(d)\right)$ is of finite $F$-dimension and so is $H^{1}\left(\mathcal{A}_{\mathrm{k}}(d)\right)$. We get $\operatorname{dim}_{\mathbb{F}} H^{0}\left(\mathcal{A}_{\mathrm{k}}(d)\right)=\operatorname{dim}_{\mathbb{F}} H^{1}\left(\mathcal{A}_{\mathrm{k}}(\mathfrak{c}-d)\right)$. So for the Euler characteristic we obtain $\chi_{\mathcal{A}_{\mathrm{k}}}(d)=\operatorname{dim}_{\mathbb{F}} H^{0}\left(\mathcal{A}_{\mathrm{k}}(d)\right)-\operatorname{dim}_{\mathbb{F}} H^{1}\left(\mathcal{A}_{\mathrm{k}}(d)\right)=\chi_{\mathcal{A}_{\mathrm{k}}}(\mathfrak{c}-d)$.

We will use the virtual dimension of two $\mathbb{F}$-commensurable spaces $G, H$ (which means $G \cap H$ is of $\mathbb{F}$-finite codimension in each of them $), \operatorname{dim}_{\mathbb{F}}(G: H)=\operatorname{dim}_{\mathbb{F}} G /(G \cap H)-$ $\operatorname{dim}_{\mathbb{F}} H /(G \cap H)$. Noting it is additive on short exact sequences and comparing $\mathcal{A}_{\mathrm{k}}(d)$ and $\mathcal{A}_{\mathrm{k}}(0)$, we obtain $\operatorname{deg}_{\mathbb{F}} d=\operatorname{dim}_{\mathbb{F}}\left(\mathbb{A}_{\mathrm{k}}(d): \mathbb{A}_{\mathrm{k}}(0)\right)=\chi_{\mathcal{A}_{\mathrm{k}}}(d)-\chi_{\mathcal{A}_{\mathrm{k}}}(0)$.

Using the two formulas

$$
\operatorname{deg}_{\mathbb{F}} d=\chi_{\mathcal{A}_{\mathrm{k}}}(d)-\chi_{\mathcal{A}_{\mathrm{k}}}(0), \quad \operatorname{dim}_{\mathbb{F}} H^{0}\left(\mathcal{A}_{\mathrm{k}}(d)\right)=\operatorname{dim}_{\mathbb{F}} H^{1}\left(\mathcal{A}_{\mathrm{k}}(\mathfrak{c}-d)\right)
$$

we get

$-\operatorname{deg}_{\mathbb{F}} d=\operatorname{dim}_{\mathbb{F}} H^{0}\left(\mathcal{A}_{\mathrm{k}}(0)\right)-\operatorname{dim}_{\mathbb{F}} H^{0}\left(\mathcal{A}_{\mathrm{k}}(\mathfrak{c})\right)-\operatorname{dim}_{\mathbb{F}} H^{0}\left(\mathcal{A}_{\mathrm{k}}(d)\right)+\operatorname{dim}_{\mathbb{F}} H^{0}\left(\mathcal{A}_{\mathrm{k}}(\mathfrak{c}-d)\right)$.

We derive $\operatorname{dim}_{\mathbb{F}} H^{0}\left(\mathcal{A}_{\mathrm{k}}(d)\right)=\operatorname{dim}_{\mathbb{F}} H^{0}\left(\mathcal{A}_{\mathrm{k}}(\mathfrak{c}-d)\right)+\operatorname{deg}_{\mathbb{F}} d+\chi_{\mathcal{A}_{\mathrm{k}}}(0)$, the adelic RiemannRoch theorem. If $C$ is geometrically irreducible then $\operatorname{dim}_{\mathbb{F}} H^{0}\left(\mathcal{A}_{\mathrm{k}}(0)\right)=1$ and $\chi_{\mathcal{A}_{\mathrm{k}}}(0)=$ $1-g$ where $g$ is the genus $\operatorname{dim}_{\mathbb{F}} H^{1}\left(\mathcal{A}_{\mathrm{k}}(0)\right)$.

It is not difficult to see that the adelic cohomology spaces $H^{i}\left(\mathcal{A}_{\mathrm{k}}(d)\right)$ are isomorphic to the Zariski cohomology spaces $H^{i}\left(C, \mathcal{O}_{C}(d)\right)$ and then the previous equality gives the classical Riemann-Roch theorem.

A differential map in the terminology of [A, Ch.XIII §4]) is a continuous linear map from $\mathbb{A}_{k}$ to $\mathbb{F}$ which vanishes on $k$. One easily shows that the space of differential maps is algebraically and topologically isomorphic to the space $\Omega_{\mathrm{k} / \mathbb{F}}^{1}$. Moreover, one can start with differential maps, without using differential forms, prove that they form a one-dimensional space over k, e.g. [A, Ch.XIII], and apply them to derive all the results in this section.

Above we used several times the classical argument to deduce finiteness of $\mathbb{F}$-dimension by establishing discreteness and linear compactness. The same argument will be applied later in the proof of the theorem in section 3. 
This proof is presented in such a way that it immediately further extends to a projective integral curve $C$ over a perfect field $\mathbb{F}([\mathrm{G}])$. The adelic object $\mathbb{A}_{C}(0)$ is defined as the product of the completions of the local rings of $C$ at its closed points, it is an order of $\mathbb{A}_{\mathrm{k}}, \mathrm{k}$ is the function field of $C$. For every Cartier divisor $d$ on $C$ one defines $\mathbb{A}_{C}(d)$ similarly.

The adelic complex for $C$ is

$$
\mathcal{A}_{C}(d): \quad \mathrm{k} \oplus \mathbb{A}_{C}(d) \longrightarrow \mathbb{A}_{\mathrm{k}}
$$

quasi-isomorphic to the complexes $\mathbb{A}_{C}(d) \longrightarrow \mathbb{A}_{\mathrm{k}} / \mathrm{k}, \mathrm{k} \longrightarrow \mathbb{A}_{\mathrm{k}} / \mathbb{A}_{C}(d)$. Using the differential pairing for $\mathbb{A}_{k}$ and the associated duality one similarly derives the Riemann-Roch theorem. In particular, for Gorenstein curves (e.g. curves lying on smooth surfaces) the complement $\mathbb{A}_{C}(d)^{\perp}$ of $\mathbb{A}_{C}(d)$ with respect to the differential pairing is $\mathbb{A}_{C}(\mathfrak{c}-d)$ where $\mathbb{A}_{C}(\mathfrak{c})=\mathbb{A}_{C}(0)^{\perp}$. Similarly to the case of smooth curves, $\operatorname{deg}_{\mathbb{F}} d=\chi_{\mathcal{A}_{C}}(d)-\chi_{\mathcal{A}_{C}}(0)$ and $\operatorname{dim}_{\mathbb{F}} H^{0}\left(\mathcal{A}_{C}(d)\right)=\operatorname{dim}_{\mathbb{F}} H^{1}\left(\mathcal{A}_{C}(\mathfrak{c}-d)\right)$, hence $\operatorname{dim}_{\mathbb{F}} H^{0}\left(\mathcal{A}_{C}(d)\right)=\operatorname{dim}_{\mathbb{F}} H^{0}\left(\mathcal{A}_{C}(\mathfrak{c}-\right.$ $d))+\operatorname{deg}_{\mathbb{F}} d+\chi_{\mathcal{A}_{C}}(0)$. If $C$ is geometrically irreducible then $\chi_{\mathcal{A}_{C}}(0)=1-g^{\prime}$ where $g^{\prime}=\operatorname{dim}_{\mathbb{F}} H^{1}\left(\mathcal{A}_{C}(0)\right)=g+\delta, g=\operatorname{dim}_{\mathbb{F}} H^{1}\left(\mathcal{A}_{\mathrm{k}}(0)\right), \delta=\operatorname{dim}_{\mathbb{F}}\left(\mathbb{A}_{\mathrm{k}}(0): \mathbb{A}_{C}(0)\right)$.

1. Let $S$ be a proper smooth irreducible surface over a perfect field $\mathbb{F}$, hence projective. Denote by $K$ its function field and assume that no nontrivial finite extension of $\mathbb{F}$ is contained in $K$. For an irreducible proper curve $y$ (we will often call them just curves) on $S$ we denote by $D_{y}$ the divisor of $y$ and by $K_{y}$ the fraction field of the completion $\mathcal{O}_{y}$ of the local ring at $y$. For a closed point $x$ of $S$ we denote by $\mathcal{O}_{x}$ the completion of the local ring at $x$.

For a closed point $x \in y$ consider the localisation of $\mathcal{O}_{x}$ at the local equation of $y$ and complete it with respect to the intersection of its maximal ideals, denote the result by $\mathcal{O}_{x, y}$ and let $K_{x, y}$ be its quotient ring. The ring $\mathcal{O}_{x, y}$ (resp. $K_{x, y}$ ) is isomorphic to the product of all $\mathcal{O}_{x, z}$ (resp. $K_{x, z}$ ) where $z$ runs through all minimal prime ideals of the completion of the local ring of $y$ at $x$, i.e. through all formal branches $y(x)$ of $y$ at $x$. The ring $\mathcal{O}_{x, z}$ is the ring of integers with respect to the discrete valuation of rank 1 on $K_{x, z}$. Denote its residue field by $E_{x, z}$, which is a one-dimensional local field and denote its residue field, a perfect field, by $k_{z}(x)$. One can also describe how to get the ring $\mathcal{O}_{x, y}$ from the ring $\mathcal{O}_{y}$ using the closed point $x \in y$, the procedure being a two-dimensional version of taking the completion of $k(y)$ with respect to $x$, see [KS]. See [F4, §24] and [M3, sect. 7-8] for more information. Choosing a local parameter $t$ of $K_{x, z}$ with respect to its discrete valuation of rank 1 , for example a local parameter $t_{y}$ of $y$ on $S$, the field $K_{x, z}$ can be viewed as the formal power series field $E_{x, z}((t))$.

Denote by $K_{x}$ be the minimal subring of $K_{x, y}$ which contains $K$ and $\mathcal{O}_{x}$. We have $K \mathcal{O}_{y}=K_{y}, K \mathcal{O}_{x}=K_{x}$.

For every curve $y$ on $S$ define the space $\mathbf{O A}_{y}$ as consisting of elements $\left(\alpha_{x, y}\right) \in$ $\prod_{x \in y} \mathcal{O}_{x, y}$ such that for every $m>0$ for almost all $x \in y$ the element $\alpha_{x, y}$ is in $\mathcal{O}_{x}+\mathcal{M}_{y}^{m}$, where $\mathcal{M}_{y}$ is the maximal ideal of $\mathcal{O}_{y}$. Define $\mathbf{A}_{y}$ as the minimal subring of $\prod_{x \in y} K_{x, y}$ which contains $K$ and $\mathbf{O A} \mathbf{A}_{y}$. Put $\mathbf{A}_{y}^{r}=t_{y}^{r} \mathbf{O} \mathbf{A}_{y}=\mathcal{M}_{y}^{r} \mathbf{O} \mathbf{A}_{y}$. This definition is equivalent to the definition of $\mathbf{A}_{y}=\cup \mathbb{A}_{y}^{r}$ given in [F4, §25] if $\mathbb{F}$ is finite. The space $\mathbf{A}_{y}$ is the two-dimensional adelic commutative algebra associated to the curve $y$ on the surface 
$S$. One way to think about $\mathbf{A}_{y}$ as $\mathbb{A}_{k(y)}\left(\left(t_{y}\right)\right)$, the formal power series in $t_{y}$ which is a local parameter of $y$ on $S$, over the adelic space of the one-dimensional function field of $y$ ( $\mathbf{A}_{y}$ does not depend on the choice of the formal variable $t_{y}$, due to the previous description), then $\mathbf{O A}_{y}=\mathbf{A}_{y}^{0}=\mathbf{A}_{y} \cap \prod_{x \in y} \mathcal{O}_{x, y}=\mathbb{A}_{k(y)}\left[\left[t_{y}\right]\right]$.

Similarly to $[\mathrm{F} 4, \S 28]$ define the geometric adelic space $\mathbf{A}_{S}$ as the restricted product of $\mathbf{A}_{y}$ with respect to $\mathbf{O} \mathbf{A}_{y}$. Equivalently, $\mathbf{A}=\mathbf{A}_{S}$ is the subalgebra of all $\left\{\left(\alpha_{x, z}\right), \alpha_{x, z} \in\right.$ $\left.\left.K_{x, z}\right)\right\}$ such that the following two restricted conditions are satisfied: for almost every $y$ the element $\alpha_{x, y} \in \mathcal{O}_{x, y}$ for all $x \in y$ and there is $r$ such that $\left(\alpha_{x, y}\right)_{x \in y} \in \mathbf{A}_{y}^{r}$ for all $y$. The adelic object $\mathbf{A}$ equals $A_{012}$ defined in [P2, §2.1]. The space $\mathbf{A}$ is the union of subspaces $\mathbf{A}(D)$ where $D$ runs through all 1-cycles (divisors) of $S$ and $\mathbf{A}(D)=\left\{\left(\alpha_{x, y}\right)_{x \in y} \in \mathbf{A}_{y}^{r_{y}}\right.$ for all $\left.y\right\}$ where $r_{y}=-v_{y}(D), D=\sum-r_{y} D_{y}$.

The space $\mathbf{A}$ is a subset of $\prod_{x \in y} K_{x, y}$. Denote

$$
\begin{aligned}
& \mathbf{B}=\prod K_{y} \cap \mathbf{A}, \quad \mathbf{C}=\prod K_{x} \cap \mathbf{A}, \\
& \mathbf{O A}=\prod \mathcal{O}_{x, y} \cap \mathbf{A}, \quad \mathbf{O B}=\prod \mathcal{O}_{y} \cap \mathbf{A}=\prod \mathcal{O}_{y}, \quad \mathbf{O C}=\prod \mathcal{O}_{x} \cap \mathbf{A}=\prod \mathcal{O}_{x} .
\end{aligned}
$$

the intersections are taken inside $\prod K_{x, z}$.

We have the diagrams of the spaces
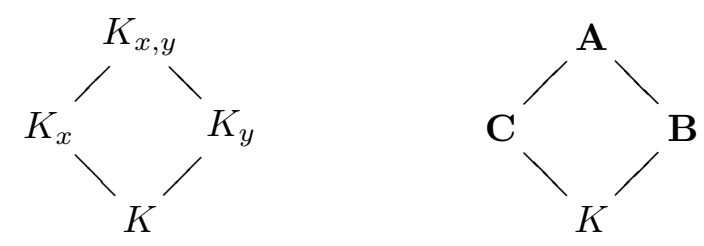

We call the adelic spaces in bold font two-dimensional full geometric adeles or simply adeles. The reason why we use the bold font for these geometric adelic objects is that the bbb-notation is employed for the second adelic structure of analytic adelic objects $\mathbb{A}, \mathbb{B}$ on relative surfaces in [F4]. In dimension one the geometric and analytic adelic structures are the same.

See [B1], [Y], [F4, §28], [M2], [M3] for more general definitions of geometric adelic objects.

Following [P1], [B1], [P2], in dimension two the geometric adelic complex in the equal characteristic case is

$$
\mathcal{A}_{S}=\mathcal{A}_{S}(0): \quad K \oplus \mathbf{O B} \oplus \mathbf{O C} \rightarrow \mathbf{B} \oplus \mathbf{C} \oplus \mathbf{O A} \rightarrow \mathbf{A} .
$$

In more compatible with the underlying symplectic structure on flags of scheme points of $S$ and more convenient for computations notation we can write it as

$$
\mathcal{A}_{S}=\mathcal{A}_{S}(0): \quad A_{0} \oplus A_{1} \oplus A_{2} \longrightarrow A_{01} \oplus A_{02} \oplus A_{12} \longrightarrow A_{012}
$$

The adelic complexes for a 1 -cocycle $D$ is

$$
\mathcal{A}_{S}(D): \quad A_{0} \oplus A_{1}(D) \oplus A_{2}(D) \longrightarrow A_{01} \oplus A_{02} \oplus A_{12}(D) \longrightarrow A_{012}
$$


where $A_{*}(D)=A_{0 *} \cap \mathbf{A}(D)$. The maps are $\left(a_{0}, a_{1}, a_{2}\right) \mapsto\left(a_{0}-a_{1}, a_{2}-a_{0}, a_{1}-a_{2}\right)$ and $\left(a_{01}, a_{02}, a_{12}\right) \mapsto a_{01}+a_{02}+a_{12}$. Note that there is a misprint in the definition of $A_{02}$ in $[\mathrm{P} 1]$.

Define two-dimensional rational geometric adeles $\mathbf{A}^{\mathrm{rt}}$ as the intersection of $\prod_{x \in y} K$ with $\mathbf{A}$ inside $\prod_{x \in y} K_{x, y}$, they are studied and applied in [P1] as a two-dimensional generalisation of the classical study described in [S]. Put $\mathbf{B}^{\mathrm{rt}}=\mathbf{B} \cap \mathbf{A}^{\mathrm{rt}}, \mathbf{C}^{\mathrm{rt}}=\mathbf{C} \cap \mathbf{A}^{\mathrm{rt}}$.

Topology. Now we define an appropriate linear topology on A. The topology we use below on $\mathbf{A}$ will have more open subspaces than that which is used in [F4, Ch.1].

The field $\mathbb{F}$ is endowed with the discrete topology, fields $E_{x, z}$ are endowed with the topology of a local field, i.e. with the discrete valuation topology.

There are several approached to work with relevant topologies defined on higher local fields, e.g. [Y], [Z], [F1]. Among them the strongest is the sequential saturation of each of them. Each of them has the same set of open $\mathbb{F}$-subspaces, so for the purposes of this paper they all give the same output.

Using a subfield $E_{x, z}^{\prime}$ of $\mathcal{O}_{x, z}$ which maps isomorphically onto $E_{x, z}=\mathcal{O}_{x, z} / \mathcal{M}_{x, z}$ with respect to the residue map, where $\mathcal{M}_{x, z}$ is the maximal ideal of $\mathcal{O}_{x, z}$, the translation invariant linear topology on equal characteristic two-dimensional local fields, in line with [Z] and [F1], has the following open subspaces: $\left\{\sum a_{i} t_{y}^{i}: a_{i} \in G_{i}\right\}$ where $G_{i}$ are open subspaces of local fields $E_{x, z}^{\prime}$ such that $G_{i}=E_{x, z}^{\prime}$ for all sufficiently large $i$. This topology is the topology of the linear inductive limit of $t_{y}^{r} \mathcal{O}_{x, z}$ which have the linear projective limit of the topologies on $t_{y}^{r} \mathcal{O}_{x, z} / t_{y}^{r} \mathcal{O}_{x, z} \mathcal{M}_{x, z}^{m}$ homeomorphic to the direct sum of finitely many copies of $E_{x, z}^{\prime}$. This topology does not depend on the choice of a uniformizer of $\mathcal{O}_{x, z}$. It does not depend on the choice of the subfield $E_{x, z}^{\prime}$ if the latter is of positive characteristic, while it does depend in characteristic zero unless $E_{x, z}^{\prime}$ is algebraic over $\mathbb{Q}$. Using the geometric origin of the field $K_{x, z}$, endow it with its canonical linear topology in all characteristics: an open base of $\mathcal{O}_{x, z} / \mathcal{N}_{x, z}^{m}$ is $a\left(\mathcal{O}_{x}+\mathcal{N}_{x, z}^{m}\right)$, a runs through regular elements of $\mathcal{O}_{x, z} / \mathcal{N}_{x, z}^{m}$, the topology of $\mathcal{O}_{x, z}$ is the linear projective limit of the topologies on $\mathcal{O}_{x, z} / \mathcal{M}_{x, z}^{m}$ and the topology of $K_{x, z}$ is the linear inductive limit of $b \mathcal{O}_{x, z}$ with the scaled topology of $\mathcal{O}_{x, z}, b \in K_{x, z}$.

Recall that even if $\mathbb{F}$ is finite, two-dimensional local fields are not locally compact with respect to these topology. See also [C2] for a new recent characterisation of the topology on two-dimensional local fields, which uses bornology.

The topology on $\mathbf{A}_{y}$ and $\mathbf{A}_{y}^{r}$ are the induced topology of the product topology on $\prod_{x \in y} K_{x, y}$. The topology of $\mathbf{A}_{y}^{r}$ is the linear projective limit topology of the topology on $\mathbf{A}_{y}^{r} / \mathcal{M}_{y}^{m} \mathbf{A}_{y}^{r}, m \geqslant 1$. The topology on $\mathbf{A}_{y}^{r}$ coincides with the linear topology in which subspaces $t_{y}^{r}\left(\prod_{x \in y} \prod_{z \in y(x)} W_{x, z}+\mathcal{M}_{y}^{m} \mathbf{A}_{y}\right), m \geqslant 1$, form an open base of 0 . Here $W_{x, z}$ are open subspaces of $\mathcal{O}_{x, z}$ containing $\mathcal{M}_{y}^{m} \mathcal{O}_{x, z}$ and almost every $W_{x, z}$ is equal to $\mathcal{O}_{x}+\mathcal{M}_{y}^{m} \mathcal{O}_{x, z}$. The topology of $\mathbf{A}_{y}$ is the linear inductive limit of the topologies on $\mathbf{A}_{y}^{r}$ which are its closed subspaces. When viewing $\mathbf{A}_{y}^{r}$ as $t_{y}^{r} \mathbb{A}_{k(y)}\left[\left[t_{y}\right]\right]$, the latter is isomorphic, via the coefficients of powers of $t_{y}$, to $\prod_{i=r}^{\infty} \mathbb{A}_{k(y)}$ endowed with the product topology of the topological spaces of the one-dimensional adeles. 
The topology of the restricted product $\prod_{i \in I}^{\prime} G_{i}$ of linear topological spaces $G_{i}$ with respect to their closed subspaces $H_{i}$ is defined as the linear inductive limit topology of $G=\lim _{\longrightarrow} G_{J}, G_{J}=\prod_{i \in J} G_{i} \prod_{i \notin J} H_{i}$ endowed with the product topology, $J$ runs through finite subsets of $I$.

Applying to $G_{y}=\mathbf{A}_{y}, H_{y}=\mathbf{A}_{y}^{0}$, we get the linear topology on $\mathbf{A}$ which coincides with the induced topology from the product topology on $\prod_{x \in y} K_{x, y}$. The space $\mathbf{A}$ is complete and is not locally compact. Its definition shows that it is the iterated linear inductive and projective limits of topologies of objects of smaller dimension, which is also how the topology defined in [Y, 3.2].

Each of $\mathbb{F}$-subspaces of the participating objects of $\mathcal{A}_{S}(D)$, including $A_{*}, A_{*}(D)$, is endowed with the induced topology. The induced topology on $K_{y}$ is the topology defined similar to the topology on $\mathbf{A}_{y}$ but using functions $k(y)$ endowed with the discrete topology instead of adeles $\mathbb{A}_{k(y)}$ with their topology. We can view $K_{x}$ as a subspace of $\mathbf{A}$ by adding 0 s at $x^{\prime} \neq x$, then the induced topology on $K_{x}$ is the linear inductive limit of the topologies on $a \mathcal{O}_{x}, a \in K$, where the topology of $\mathcal{O}_{x}$ is the linear projective limit topology of the discrete topologies on $\mathcal{O}_{x} / \mathcal{M}_{x}^{n}, \mathcal{M}_{x}$ is the maximal ideal of $\mathcal{O}_{x}$.

We deduce that $\mathbf{A}(D)=\prod \mathbf{A}_{y}^{-v_{y}(D)}$ is a closed (but not open) subspace of $\mathbf{A}$ and has the topology of the direct product of $\mathbf{A}_{y}^{-v_{y}(D)}$. It is easy to see that the topology on $\mathbf{A}$ is the linear inductive limit topology of $\mathbf{A}(D)$. The topology on $\mathbf{A}(D)$ is also the linear projective limit topology of $\mathbf{A}(D) / \mathbf{A}\left(D^{\prime}\right)$ where $D^{\prime}$ runs through 1-cycles $\leqslant D$ and $\mathbf{A}(D) / \mathbf{A}\left(D^{\prime}\right) \simeq \prod \mathbf{A}_{y}^{-v_{y}(D)} / \mathbf{A}_{y}^{-v_{y}\left(D^{\prime}\right)}$ endowed with the finite product topology of the one-dimensional topologies on $\mathbb{A}_{k(y)}$.

We endow the spaces of rational adeles with the induced topology from $\mathbf{A}$. It is easy to see that $K$ is dense in $K_{y}, K_{x}, K_{x, y}$, hence $\mathbf{B}^{\mathrm{rt}}, \mathbf{C}^{\mathrm{rt}}, \mathbf{A}^{\mathrm{rt}}$ are respectively dense in B, C, A.

2. For two topological linear spaces $X, Y$ denote by $\operatorname{Hom}_{\mathbb{F}}^{c}(X, Y)$ the linear space of continuous linear maps endowed with the linear-compact-to-open topology. The space $\operatorname{Hom}_{\mathbb{F}}^{c}(X, \mathbb{F})$ is called the space of $\mathbb{F}$-characters of $X$. Since $\mathbb{F}$ has discrete topology, the basis of open linear subspaces of the space $\operatorname{Hom}_{\mathbb{F}}^{c}(X, \mathbb{F})$ is formed by finite intersections of $\left\{f \in \operatorname{Hom}_{\mathbb{F}}^{c}(X, \mathbb{F}): f(K)=0\right\}$ where $K$ is a linear compact subset of $X$. Recall that if $X$ is discrete (resp. linear compact) then $\operatorname{Hom}_{\mathbb{F}}^{c}(X, \mathbb{F})$ is linear compact (resp. discrete), see e.g. [Le,II, $\S 6]$, [Ko]. We call a topological linear space self-dual if it is algebraically and topologically isomorphic to the space of its $\mathbb{F}$-characters.

There are two general constructions which extend the class of self-dual topological linear spaces occurring in geometry and number theory.

1. Let $R$ be a commutative $\mathbb{F}$-algebra. If the space $R$ is endowed with a translation invariant topology with respect to which it is a self-dual topological linear space, then the space of the formal power series ring $R((t))$, endowed with the inductive limit linear topology of the direct product topology on $t^{i} R[[t]]$ using the topology of $R$ similarly to the previous section, is also self-dual. To show algebraic self-duality, fix a nontrivial $\mathbb{F}$-character $\psi_{0}$ from $R((t))$ to $\mathbb{F}$ which has conductor $R[[t]]$. Let an $\mathbb{F}$-character $\psi$ of $R((t))$ have conductor $t^{i} R[[t]]$, i.e. $i$ is the minimal such that $\psi\left(t^{i} R[[t]]\right)=0$. Then the 
restriction of $\psi$ on $t^{i-1} R[[t]]$ induces an $\mathbb{F}$-character on $R$, so there is an $a_{0} \in R$ such that $\psi_{1}(\alpha)=\psi(\alpha)-\psi_{0}\left(a_{0} t^{-i} \alpha\right)$ is trivial on $t^{i-1} R[[t]]$; similarly by induction, for $j \geqslant 1$ there is $a_{j} \in R$ such that $\psi_{j+1}(\alpha)=\psi_{j}(\alpha)-\psi_{0}\left(a_{j} t^{-i+j} \alpha\right)$ is trivial on $t^{i-1-j} R[[t]]$; we conclude that $\psi(\alpha)=\psi_{0}(a \alpha)$ with $a=\sum_{j=0}^{+\infty} a_{j} t^{-i+j}$. It is easy to check that for every open subspace $U$ and a linear compact subset $C$ there is an open subspace $V$ such that $V C \subset U$ (use that $C$ is contained in some $t^{i} R[[t]]$ ). This implies that the map $a \mapsto\left(\alpha \mapsto \psi_{0}(a \alpha)\right)$ is a non-canonical homeomorphism between the linear topological spaces $R((t))$ and $\operatorname{Hom}_{\mathbb{F}}^{c}(R((t)), \mathbb{F})$, it depends on the choice of $\psi_{0}$.

In particular, for every equal characteristic two-dimensional local field $F$ with last residue field $\mathbb{F}$, e.g. $K_{x, z}$, the linear topological space $F$ is (non-canonically) self-dual (see also [F2, Lemma 3]).

Similarly, the linear topological space $\mathbf{A}_{y}$ is (non-canonically) self-dual.

2. The second general construction is of restricted products. Vaguely speaking, if topological spaces $G_{i}$ are self-dual, then for a certain choice of their closed subspaces $H_{i}$ satisfying natural conditions, the restricted product of $G_{i}$ with respect to $H_{i}$ is self-dual. For a concrete application of this principle see (2) of the next theorem.

A differential map is defined for a form $\omega \in \Omega_{K / \mathbb{F}}^{2}$ as

$$
d_{\omega}: \mathbf{A} \longrightarrow \mathbb{F}, \quad\left(\alpha_{x, z}\right) \mapsto \sum_{x \in y} \operatorname{Tr}_{k(x) / \mathbb{F}}\left(\sum_{z \in y(x)} \operatorname{Tr}_{x, z} \operatorname{res}_{x, z}\left(\alpha_{x, z} \omega\right)\right) .
$$

Here $y$ runs through all proper irreducible curves on $S, x$ runs through all closed points of $y$. The map $\operatorname{res}_{x, z}: \Omega_{K_{x, z}} \rightarrow k_{z}(x)$ is the two-dimensional local residue which takes the coefficient of $t_{2}^{-1} t_{1}^{-1} d t_{2} \wedge d t_{1}$ where $t_{2}, t_{1}$ are local parameters of $K_{x, z}$, this does not depend on their choice. The map $\operatorname{Tr}_{x, z}: k_{z}(x) \rightarrow k(x)$ is the trace to the residue field $k(x)$ of $\mathcal{O}_{x}$. The adelic condition immediately implies that for each element of $\mathbf{A}$ there are only finitely many non-zero terms in the sum. See [P1], [P2], [Y], [HY1], [HY2], [F4,§27-§29], [M1], [M2] for more detail about basic properties of $d_{\omega}$ and its generalizations. There is an omission in [P1] and [OP2]: the trace maps maps $\operatorname{Tr}_{x, z}$ do not appear there in the definition of the map $d_{\omega}$ at singular points $x \in y$; however, it is straightforward to extend the arguments on [P1] to this general situation.

If $\omega$ is a non-zero form denote by $\mathfrak{C}$ its divisor, its class in $\operatorname{Pic}(S)$ is uniquely determined. The composite of the multiplication $\mathbf{A} \times \mathbf{A} \longrightarrow \mathbf{A}$ and $d_{\omega}$ gives the differential pairing

$$
\mathbf{A} \times \mathbf{A} \longrightarrow \mathbf{A} \longrightarrow \mathbb{F}, \quad(\alpha, \beta) \mapsto d_{\omega}(\alpha \beta)
$$

For a subspace $B$ of $\mathbf{A}$ denote by $B^{\perp}=\left\{\gamma \in \mathbf{A}: d_{\omega}(B \gamma)=0\right\}$ the subspace which complements $B$ with respect to $d_{\omega}$.

Define, similarly to the one-dimensional case, e.g. [A, Ch.XIII, §4], a differential map as a linear continuous homomorphism from $\mathbf{A}$ to $\mathbb{F}$, which vanishes on the lower level $A_{01}+A_{02}=\mathbf{B}+\mathbf{C}$.

THEOREM. 
(1) The differential pairing is symmetric, continuous and non-degenerate.

(2) Every $\mathbb{F}$-character of $\mathbf{A}$ is equal to $\beta \mapsto d_{\omega}(\alpha \beta)$ for a uniquely determined adele $\alpha \in \mathbf{A}$. The space $\mathbf{A}$ is (non-canonically) self-dual. In addition, if $\mathbb{F}$ is finite then every character of the additive group of $\mathbf{A}$ is equal to $\beta \mapsto \operatorname{Tr}_{\mathbb{F} / \mathbb{F}_{p}} d_{\omega}(\alpha \beta)$ composed with a fixed nontivial homomorphism $\mathbb{F}_{p} \rightarrow \mathbb{C}^{\times}$, for a uniquely determined adele $\alpha \in \mathbf{A}$.

(3) For every subspace its complement is a closed subspace. For every closed subspace $B$ of $\mathbf{A}$ we have $\left(B^{\perp}\right)^{\perp}=B$ and $B$ is isomorphic algebraically and topologically to the linear space of continuous linear maps from $\mathbf{A} / B^{\perp}$ to $\mathbb{F}$. For two subspaces $B, C$ we have $(B+C)^{\perp}=B^{\perp} \cap C^{\perp}$. For two closed subspaces $B, C$ we have $(B \cap C)^{\perp}=B^{\perp}+C^{\perp}$ and $B+C$ is closed.

(4) We have $A_{12}(D)^{\perp}=A_{12}(\mathfrak{C}-D), A_{01}^{\perp}=A_{01}, A_{02}^{\perp}=A_{02} . A_{01}, A_{02}, A_{12}$ are closed subspaces.

(5) $A_{i}=A_{i j} \cap A_{i k}$ for every $0 \leqslant i \leqslant 2$, where $i, j, k$ is a permutation of $0,1,2$ and we set $A_{i j}=A_{j i}$.

(6) Each of $A_{*}, A_{*}(D)$ and any of their sums is closed in $\mathbf{A}$

$A_{0}^{\perp}=A_{01}+A_{02}, A_{1}(D)^{\perp}=A_{01}+A_{12}(\mathfrak{C}-D), A_{2}(D)^{\perp}=A_{02}+A_{12}(\mathfrak{C}-D)$.

(7) The $K$-space of differential maps on $\mathbf{A}$ is isomorphic to the 1-dimensional $K$-space of differential forms $\left\{d_{\omega}: \omega \in \Omega_{K / \mathbb{F}}^{2}\right\}$.

Proof. Continuity and non-degenerate property follow immediately from the definitions. To construct $\alpha$ in (2), use a natural modification of the classical argument in [T, Lemma 3.2.1, Lemma 3.2.2], namely, restrict the character on $y$, find an appropriate $\alpha_{y}$, then $\alpha=\left(\alpha_{y}\right) \in \mathbf{A}$, since the character vanishes on almost all $\mathbf{O A}_{y}$. An argument similar to [T, Th. 3.2.1] implies that $\mathbf{A}$ is self-dual with respect to the pairing $\mathbf{A} \times \mathbf{A} \rightarrow \mathbb{F}$. Proof of (3) follows the classical argument, e.g. in [Le,II, $§ 5]$.

(4) The first equality follows from the definitions. The property $A_{01} \subset A_{01}^{\perp}$ is the reciprocity law on curves, the way how it is proved for rational geometric adeles in [P1], by reducing to the one-dimensional case, extends immediately to full geometric adeles, and so does the proof of the opposite inclusion.

The inclusion $A_{02} \subset A_{02}^{\perp}$ is the reciprocity law for points and its proof is reduced to one point $x$. Multiplying $\omega$ by a regular element of $K_{x}$ one can assume that $\omega$ near $x$ is $d t \wedge d u$, where $t, u$ are generators of local equations of two global curves $y_{1}, y_{2}$ with transversal intersection, and $\mathcal{O}_{x}=k(x)[[u, t]]$. Then the proof for adeles is an easy verification, or use a natural extension of the argument for rational adeles in [P1]. For the opposite inclusion one can use a two-dimensional version of the classical argument in [W, Ch.IV§2] as follows. We have $\mathcal{O}_{x, y_{1}}=k(x)((u))[[t]], K_{x, y_{1}}=k(x)((u))((t))=$ $\mathcal{O}_{x, y_{1}}+t^{-1} k(x)[[u]]\left[t^{-1}\right]+t^{-1} u^{-1} k(x)\left[t^{-1}, u^{-1}\right]$. For every curve $y \neq y_{1}, x \in y_{1}$, put $R_{y}=\mathcal{O}_{x, y}$, and put $R_{y_{1}}=u^{-1} k(x)\left[u^{-1}\right][[t]]+t^{-1} u^{-1} k(x)\left[t^{-1}, u^{-1}\right]$, denote $R=\prod_{y} R_{y}$. Note that $R_{y_{1}} R_{y_{1}} \subset u^{-2} k(x)\left[u^{-1}\right]((t))$. We have $\mathcal{O}_{x, y_{1}}=\mathcal{O}_{x}+u^{-1} k(x)\left[u^{-1}\right][[t]]$, so $R+\mathcal{O}_{x}=R^{\prime}=R_{y_{1}}^{\prime} \times \prod_{y \neq y_{1}} R_{y}$ where $R_{y_{1}}^{\prime}=\mathcal{O}_{x, y_{1}}+t^{-1} u^{-1} k(x)\left[t^{-1}, u^{-1}\right]$. The space $t^{-1} k(x)[[u]]\left[t^{-1}\right]$ is a subspace of $K_{x} \cap \cap_{y \neq y_{1}} R_{y}$ and its sum with $R_{y_{1}}^{\prime}$ gives $K_{x, y_{1}}$, so we get $R_{y_{1}}^{\prime}+K_{x} \cap \cap_{y \neq y_{1}} R_{y}=K_{x, y_{1}}$ and so $R+K_{x}=R^{\prime}+K_{x} \supset K_{x, y_{1}} \times \prod_{y \neq y_{1}} \mathcal{O}_{x, y}$. 
Similarly to [P1, p. 710] apply the strong approximation theorem ([Bo, Ch. VII $\S 5]$ ) to $\operatorname{Spec}\left(\mathcal{O}_{x}\right) \backslash \operatorname{Spec}\left(\mathcal{O}_{x}\right) \cap y_{1}$ to deduce that the sum of $K_{x, y_{1}} \times \prod_{y \neq y_{1}} \mathcal{O}_{x, y}$ and the quotient field $K_{x}^{\prime}$ of $\mathcal{O}_{x}$ equals $\mathbf{A}_{x}$, the $x$-part of $\mathbf{A}$. Since $K_{x}^{\prime}+R^{\prime}=K_{x}+R^{\prime}$, we deduce $R+K_{x}=\mathbf{A}_{x}$. Now let $\alpha \in \mathbf{A}_{x} \cap K_{x}^{\perp}$. Write $\alpha=a+\beta$ with $a \in K_{x}, \beta \in R$. Then $\beta \in K_{x}^{\perp}$. From $d_{\omega}(R R)=0$ we obtain $\beta \in \mathbf{A}_{x}^{\perp}$. Thus, using (1) we get $\beta=0, \alpha \in K_{x}$.

The other properties follow, using (3).

(5) The property for $i=1,2$ follows from the definitions. To prove the property for $i=0$, i.e. to show that $K$ equals to the $K$-space $\mathbf{B} \cap \mathbf{C}$, the following argument looks the shortest. The previous parts of the theorem imply that the $K$-space $\mathbf{B} \cap \mathbf{C}$ is algebraically and topologically isomorphic to the $K$-space $\operatorname{Hom}_{\mathbb{F}}^{c}(\mathbf{A} /(\mathbf{B}+\mathbf{C}), \mathbb{F})$. The kernel of every element of the latter space is an open subspace of $\mathbf{A}$, whose sum with rational adeles $\mathbf{A}^{\text {rt }}$ equals $\mathbf{A}$. Therefore the linear map $\operatorname{Hom}_{\mathbb{F}}^{c}(\mathbf{A} /(\mathbf{B}+\mathbf{C}), \mathbb{F}) \rightarrow \operatorname{Hom}_{\mathbb{F}}^{c}\left(\mathbf{A}^{\mathrm{rt}} /\left(\mathbf{B}^{\mathrm{rt}}+\mathbf{C}^{\mathrm{rt}}\right), \mathbb{F}\right)$, associated to $\mathbf{A}^{\mathrm{rt}} /\left(\mathbf{B}^{\mathrm{rt}}+\mathbf{C}^{\mathrm{rt}}\right) \rightarrow \mathbf{A} /(\mathbf{B}+\mathbf{C})$, is injective. Now one can use rational adelic versions of (3) and (4), proved exactly in the same way, or $[\mathrm{P} 1, \S 2]$, to deduce that the complement of $\mathbf{B}^{\mathrm{rt}} \cap \mathbf{C}^{\mathrm{rt}}$ in $\mathbf{A}^{\mathrm{rt}}$ is $\mathbf{B}^{\mathrm{rt}}+\mathbf{C}^{\mathrm{rt}}$ and the $K$-space $\operatorname{Hom}_{\mathbb{F}}^{c}\left(\mathbf{A}^{\mathrm{rt}} /\left(\mathbf{B}^{\mathrm{rt}}+\mathbf{C}^{\mathrm{rt}}\right), \mathbb{F}\right)$ is isomorphic to the $K$-space $\mathbf{B}^{\text {rt }} \cap \mathbf{C}^{\mathrm{rt}}$. Elements of the latter do not depend on $x$ and $y$, so this space is $K$. We conclude $\mathbf{B} \cap \mathbf{C}=K$.

(6) follows from the previous.

(7) The action of $K$ on differential maps $d$ is $k * d: \alpha \mapsto d(k \alpha)$. The property follows from the previous properties.

\section{REMARKS.}

1. This theorem together with its proof does not seem to be in the published literature, but some of its statements may have appeared elsewhere. Parts of (4)-(6) of this theorem for full adeles for surfaces over finite fields were stated in [OP2, Prop.2] without proof. The topological aspects, including self-duality, seem to have not yet been published elsewhere.

2. $[\mathrm{P} 1, \S 3]$ contains a proof of (7) for rational adeles which uses a different topology on adeles in which the open base is $\mathbf{A}(D)$ for divisors $D$ (it also uses the isomorphism between the rational adelic cohomologies and Zariski cohomologies, which we try to avoid to use till almost the end of this paper). The space $\mathbf{A} /(\mathbf{B}+\mathbf{C}+\mathbf{A}(D))$ is of finite $\mathbb{F}$-dimension and hence discrete (see the theorem in the next section). An open subspace in the topology of $\mathbf{A}$ contains some $\mathbf{A}(D)$, and the space $\operatorname{Hom}_{\mathbb{F}}^{c}(\mathbf{A} /(\mathbf{B}+\mathbf{C}), \mathbb{F})$ is the inductive limit of the discrete spaces $\operatorname{Hom}_{\mathbb{F}}^{c}(\mathbf{A} /(\mathbf{B}+\mathbf{C}+\mathbf{A}(D)), \mathbb{F})=\operatorname{Hom}_{\mathbb{F}}(\mathbf{A} /(\mathbf{B}+\mathbf{C}+\mathbf{A}(D)), \mathbb{F})$, and similarly for rational adeles. Thus, the topology of the space $\operatorname{Hom}_{\mathbb{F}}^{c}\left(\mathbf{A}^{\mathrm{rt}} /\left(\mathbf{B}^{\mathrm{rt}}+\mathbf{C}^{\mathrm{rt}}\right), \mathbb{F}\right)$ in this paper is the same as its topology in $[\mathrm{P} 1, \S 3]$.

3. One can think about an even better version of the theory. Namely, similarly to the one-dimensional approach by Artin in [A, Ch.XIII], one can start with the definition of differential maps on adeles, given before the theorem, and without using differential forms and their properties (a) prove existence of differential maps on adeles, (b) establish that they form a one-dimensional space over $K$, and then derive all the results in this paper.

The notion of differential map is also related to a more general notion of locally differential operator in [Y, Def 3.1.8]. 
4. Self-duality of the adelic spaces in this paper, which are not locally compact, leads to a natural question about an extension of general duality theory for linear locally compact abelian spaces to a larger class of linear topological spaces which contains those appearing in higher number theory. There are already several categorical approaches ([B2], [Kt], [Kp], [Pr]), but precise relations between them and the topological point of view are not yet fully clear.

3. There are natural maps from the complex $\mathcal{A}_{S}(D)$ to the following complexes

$$
\begin{array}{ll}
\mathrm{C}_{1}(D): & A_{0} \longrightarrow A_{01} / A_{1}(D) \longrightarrow A_{012} /\left(A_{12}(D)+A_{02}\right), \\
\mathrm{C}_{2}(D): & A_{2}(D) \longrightarrow A_{12}(D) / A_{1}(D) \longrightarrow A_{012} /\left(A_{01}+A_{02}\right), \\
\mathrm{C}_{3}(D): & A_{0} \longrightarrow A_{02} / A_{2}(D) \longrightarrow A_{012} /\left(A_{12}(D)+A_{01}\right) .
\end{array}
$$

They are described as follows:

$\mathcal{A}_{S}(D) \rightarrow \mathcal{C}_{1}(D)$ is given by the projection to the first term, the projection to the first term and quotient, the quotient;

$\mathcal{A}_{S}(D) \rightarrow \mathcal{C}_{2}(D)$ is given by the minus projection to the third term, the projection to the third term and quotient, the quotient;

$\mathcal{A}_{S}(D) \rightarrow \mathcal{C}_{3}(D)$ is given by the projection to the first term, the projection to the second term and quotient, the quotient.

Let $H^{i}\left(\mathcal{A}_{S}(D)\right)$ be the cohomology spaces of the complex $\mathcal{A}_{S}(D)$. Using the previous theorem (5), it is immediate to deduce that these maps of complexes induce algebraic isomorphisms between the appropriate cohomology spaces of the complexes.

The maps from $\mathcal{A}_{S}(D)$ to the other complexes are clearly continuous and hence the algebraic isomorphisms from the cohomologies of $\mathcal{A}_{S}(D)$ to the cohomologies of $\mathcal{C}_{j}(D)$ are continuous. It is easy to see that they are also open maps, using that the projection on components is an open map.

Thus, we get algebraic and topological isomorphisms

$$
\begin{aligned}
H^{0}\left(\mathcal{A}_{S}(D)\right) & \simeq A_{0} \cap A_{12}(D)=A_{0} \cap A_{1}(D)=A_{0} \cap A_{2}(D)=A_{1}(D) \cap A_{2}(D), \\
H^{1}\left(\mathcal{A}_{S}(D)\right) & \simeq\left(A_{01} \cap\left(A_{12}(D)+A_{02}\right)\right) /\left(A_{1}(D)+A_{0}\right) \\
& \simeq\left(A_{12}(D) \cap\left(A_{01}+A_{02}\right)\right) /\left(A_{1}(D)+A_{2}(D)\right) \\
& \simeq\left(A_{02} \cap\left(A_{12}(D)+A_{01}\right)\right) /\left(A_{2}(D)+A_{0}\right), \\
H^{2}\left(\mathcal{A}_{S}(D)\right) & =A_{012} /\left(A_{12}(D)+A_{01}+A_{02}\right) .
\end{aligned}
$$

For an algebraic version of this description for the rational adeles see [P1, p. 714].

THEOREM.

(1) There are algebraic and topological isomorphisms

$$
\operatorname{Hom}_{\mathbb{F}}^{c}\left(H^{i}\left(\mathcal{A}_{S}(D)\right), \mathbb{F}\right) \simeq H^{2-i}\left(\mathcal{A}_{S}(\mathfrak{C}-D)\right) \quad \text { for } 0 \leqslant i \leqslant 2 .
$$

(2) Each $\operatorname{dim}_{\mathbb{F}} H^{i}\left(\mathcal{A}_{S}(D)\right)$ is finite and an invariant of the class of $D$ in $\operatorname{Pic}(S)$. The space $\mathbf{A} /(\mathbf{B}+\mathbf{C})$ is $\mathbb{F}$-linear compact and $K$ is discrete. 
(3) Denote by $\chi_{\mathcal{A}_{S}}(D)=\sum(-1)^{i} \operatorname{dim}_{\mathbb{F}} H^{i}\left(\mathcal{A}_{S}(D)\right)$ the Euler characteristic of the complex $\mathcal{A}_{S}(D)$. Then $\chi_{\mathcal{A}_{S}}(D)=\chi_{\mathcal{A}_{S}}(\mathfrak{C}-D)$ and is finite.

Proof. By the previous theorem

$$
H^{0}\left(\mathcal{A}_{S}(D)\right)^{\perp} \simeq\left(A_{0} \cap A_{12}(D)\right)^{\perp}=A_{01}+A_{02}+A_{12}(\mathfrak{C}-D),
$$

so the space of $\mathbb{F}$-characters of $H^{0}\left(\mathcal{A}_{S}(D)\right)$ is algebraically and topologically isomorphic to

$$
A_{012} / H^{0}\left(\mathcal{A}_{S}(D)\right)^{\perp} \simeq H^{2}\left(\mathcal{A}_{S}(\mathfrak{C}-D)\right)
$$

and the space of continuous linear maps from $H^{2}\left(\mathcal{A}_{S}(D)\right)$ to $\mathbb{F}$ is algebraically and topologically isomorphic to $H^{0}\left(\mathcal{A}_{S}(\mathfrak{C}-D)\right)$.

The space of continuous linear maps from the space $H^{1}\left(\mathcal{A}_{S}(D)\right)$, which is algebraically and topologically isomorphic to $\left(A_{01} \cap\left(A_{12}(D)+A_{02}\right)\right) /\left(A_{1}(D)+A_{0}\right)$, to $\mathbb{F}$ is algebraically and topologically isomorphic to

$$
\begin{aligned}
& \left(A_{1}(D)^{\perp} \cap A_{0}^{\perp}\right) /\left(A_{01}^{\perp}+\left(A_{12}(D)^{\perp} \cap A_{02}^{\perp}\right)\right) \\
& =\left(\left(A_{01}+A_{12}(\mathfrak{C}-D)\right) \cap\left(A_{01}+A_{02}\right)\right) /\left(A_{01}+A_{02} \cap A_{12}(\mathfrak{C}-D)\right)
\end{aligned}
$$

which is equal to

$$
\left(\left(A_{01}+A_{12}(\mathfrak{C}-D)\right) \cap\left(A_{01}+A_{02}\right)\right) /\left(A_{01}+A_{2}(\mathfrak{C}-D)\right)
$$

by the previous theorem.

We have a natural map, induced by embeddings, from

$$
\left(A_{12}(\mathfrak{C}-D) \cap\left(A_{01}+A_{02}\right)\right) /\left(A_{1}(\mathfrak{C}-D)+A_{2}(\mathfrak{C}-D)\right)
$$

to the latter and it is easy to see it is an algebraic isomorphism. It is also continuous and by the previous theorem (3) the image of a closed space is a closed space, so it is a homeomorphism. Thus, the space of $\mathbb{F}$-characters of $H^{1}\left(\mathcal{A}_{S}(D)\right)$ is algebraically and topologically isomorphic to $H^{1}\left(\mathcal{A}_{S}(\mathfrak{C}-D)\right)$.

The description of $H^{i}$ above and $A_{*}(\operatorname{Div}(f)+D)=A_{*}(D)-f$ for any $f \in K^{\times}$,* is $1,2,12$, implies that $\operatorname{dim}_{\mathbb{F}} H^{i}\left(\mathcal{A}_{S}(D)\right)$ is an invariant of the class of $D$ in $\operatorname{Pic}(S)$.

We get $H^{0}\left(\mathcal{A}_{S}(0)\right) \simeq K \cap \mathbf{O A}=\mathbb{F}$. Finiteness of $\mathbb{F}$-dimension of $H^{0}\left(\mathcal{A}_{S}(D)\right)$ will follow from finiteness of $\mathbb{F}$-dimension of $H^{0}\left(\mathcal{A}_{S}(D)\right) / H^{0}\left(\mathcal{A}_{S}\left(D-D_{y}\right)\right)$ : using the moving lemma [Li, 9.1.10], find a linearly equivalent divisor $D^{\prime}$ to $D$ whose support does not include $y$. The quotient $\left(K \cap A_{12}\left(D^{\prime}\right)\right) /\left(K \cap A_{12}\left(D^{\prime}-D_{y}\right)\right)$ is isomorphic to $k(y) \cap \mathbb{A}_{k(y)}\left(\left.D^{\prime}\right|_{y}\right)$, hence is of finite $\mathbb{F}$-dimension by $\mathbf{0}$. Due to the first isomorphism $H^{2}\left(\mathcal{A}_{S}(D)\right)$ is also of finite $\mathbb{F}$-dimension.

The quotient $\mathbf{O A}_{y} /\left(K_{y} \cap \mathbf{O A}_{y}\right) \simeq \mathbb{A}_{k(y)}\left[\left[t_{y}\right]\right] / k(y)\left[\left[t_{y}\right]\right]$ is algebraically and topologically isomorphic to the direct product of linear compact spaces $\mathbb{A}_{k(y)} / k(y)$ and hence is linear compact. Therefore the quotient $A_{12}(0) / A_{1}(0)$ and then $A_{12}(D) / A_{1}(D)$ are linear compact. Since the space $A_{12}(D) / A_{1}(D)$ is linear compact and $H^{2}\left(\mathcal{A}_{S}(D)\right)$ is of finite $\mathbb{F}$-dimension, using $\mathcal{C}_{2}(D)$ we obtain $A_{012} /\left(A_{01}+A_{02}\right)$ is linear compact. The space $A_{0}$ is algebraically and topologically isomorphic to the space of $\mathbb{F}$-characters of $A_{012} /\left(A_{01}+A_{02}\right)$, hence $A_{0}$ is discrete. The space $H^{1}\left(\mathcal{A}_{S}(D)\right)$ is algebraically and topologically isomorphic to the closed subspace of the quotient of $A_{12}(D) / A_{1}(D)$, therefore, 
$H^{1}\left(\mathcal{A}_{S}(D)\right)$ is linear compact. By $(1), H^{1}\left(\mathcal{A}_{S}(D)\right)$ is algebraically and topologically isomorphic to the space of $\mathbb{F}$-characters of the linear compact space $H^{1}\left(\mathcal{A}_{S}(\mathfrak{C}-D)\right)$, hence $H^{1}\left(\mathcal{A}_{S}(D)\right)$ is discrete. Being both discrete and linear compact, $H^{1}\left(\mathcal{A}_{S}(D)\right)$ is of finite $\mathbb{F}$-dimension.

(3) follows immediately.

REMARKS.

1. Proof of property (1) for rational adeles can be found in [P1]. (2) gives the first direct adelic proof of finite dimensionality of cohomologies of the adelic complex, without using Zariski cohomologies of $\mathcal{O}_{S}(D)$ and its properties.

2. The previous theorem gives the first adelic proof of the discreteness of $K$ in $\mathbf{A}$. The discreteness of $K$ as a topological subspace of $\mathbf{A}$ is a two-dimensional version of the well known similar fact in dimension one: the discreteness of a global field $\mathrm{k}$ as a topological subspace of the one-dimensional adeles $\mathbb{A}_{\mathrm{k}}$. The discreteness of $K$ inside $\mathbf{A}$ is used in the study of an interplay between the geometric and analytic adelic structures on elliptic surfaces in [F5] in relation to the BSD conjecture. Unlike the compactness of the quotient $\mathbb{A}_{\mathrm{k}} / \mathrm{k}$, the quotient $\mathbf{A} / K$ is not compact but the quotient $\mathbf{A} / K^{\perp}=\mathbf{A} /(\mathbf{B}+\mathbf{C})$ is.

3. The cohomology spaces $H^{i}\left(\mathcal{A}_{S}(D)\right)$ for full adeles are naturally homeomorphic to the cohomology spaces $H^{i}\left(\mathcal{A}_{S}^{\mathrm{rt}}(D)\right)$ for rational adeles via the maps induced by $\mathbf{A}^{\mathrm{rt}} \longrightarrow \mathbf{A}$. This is obvious for $i=0$ and it follows for $i=2$ due to (1) of the previous theorem and the similar statement for rational adeles. Using $\mathcal{C}_{1}$ and its rational adeles version $\mathcal{C}_{1}^{\mathrm{rt}}$, the map $f:\left(A_{01}^{\mathrm{rt}} \cap\left(A_{12}^{\mathrm{rt}}(D)+A_{02}^{\mathrm{rt}}\right)\right) /\left(A_{1}^{\mathrm{rt}}(D)+A_{0}\right) \longrightarrow\left(A_{01} \cap\left(A_{12}(D)+A_{02}\right)\right) /\left(A_{1}(D)+A_{0}\right)$ is the map between their $H^{1}$ s. Denote the numerator and denominator of the second space by $V$ and $W$. Then this map is $\left(V \cap \mathbf{A}^{\mathrm{rt}}\right) /\left(W \cap \mathbf{A}^{\mathrm{rt}}\right) \longrightarrow V / W$. The difference of the finite $\mathbb{F}$-dimensions of these spaces is the dimension of the space $\left(V+\mathbf{A}^{\mathrm{rt}}\right) /\left(W+\mathbf{A}^{\mathrm{rt}}\right)$. Hence the latter is discrete, on the other hand its denominator is dense in the numerator, hence this space is 0 and $f$ is a homeomorphism.

4. The adelic Euler characteristic $\chi_{\mathcal{A}_{S}}(D)$ is not an additive function of $D$. Define a pairing on divisors

$$
[E, D]:=\chi_{\mathcal{A}_{S}}(0)-\chi_{\mathcal{A}_{S}}(-D)-\chi_{\mathcal{A}_{S}}(-E)+\chi_{\mathcal{A}_{S}}(-D-E) .
$$

We will use defining properties of the intersection pairing to deduce that the pairing $[$, coincides with the intersection pairing $($, ).

But first, a useful natural property which relates the dimension two and dimension one theories, it is an extension of a property of rational adeles [P1, §2, Prop.3].

Lemma. Let $D_{y}$ be a prime divisor of an irreducible proper curve y on $S$. Let $D$ be a divisor on $S$ whose support does not include $y$.

For a divisor $d$ on $y$ denote by $\mathfrak{C}_{y}(d)$ the complex $\mathbb{A}_{y}(d) \longrightarrow \mathbb{A}_{k(y)} / k(y)$ in degree 0 and 1 and with 0 in degree 2, quasi-isomorphic to the complex $\mathcal{A}_{y}(d)$ from section $\mathbf{0}$. 
Using the complex $\mathfrak{C}_{2}(D)$ from section 3 , quasi-isomorphic to the complex $\mathcal{A}_{S}(D)$, we have an exact sequence of complexes

$$
0 \longrightarrow \mathcal{C}_{2}\left(D-D_{y}\right) \longrightarrow \mathcal{C}_{2}(D) \longrightarrow \mathcal{C}_{y}\left(\left.D\right|_{y}\right) \longrightarrow 0
$$

with the maps defined below in the proof.

Hence

$$
\chi_{\mathcal{A}_{S}}(D)=\chi_{\mathcal{A}_{S}}\left(D-D_{y}\right)+\chi_{\mathcal{A}_{y}}\left(\left.D\right|_{y}\right)
$$

Proof. We have a natural commutative diagram

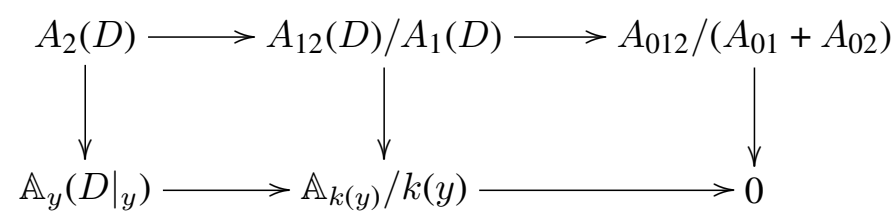

where the vertical maps are defined as follows: $A_{12}(D) \longrightarrow \mathbb{A}_{k(y)}$ is obtained via the projection to the $y$-component which gives an element of $\mathbf{O A}_{y}$, since the support of $D$ does not contain $y$, and then the projection $p_{y}$ to the residue level $\mathbf{O A}_{y} \rightarrow \mathbb{A}_{k(y)}$; $A_{1}(D) \longrightarrow k(y)$ is obtained via the projection to the $y$-component which gives an element of $\mathbf{O B}_{y}$ and then the projection to the residue field $\mathbf{O B}_{y} \rightarrow k(y) ; A_{2}(D) \longrightarrow \mathbb{A}_{y}\left(\left.D\right|_{y}\right)$ is induced by $A_{12}(D) \longrightarrow \mathbb{A}_{k(y)}$. All these maps are surjective. The interesting case is that of the map $A_{2}(D) \longrightarrow \mathbb{A}_{y}\left(\left.D\right|_{y}\right)$. Its surjectivity follows from an adelic description of the local multiplicity. Namely, for $x \in y$ the $x$-part $p_{y, x}$ of the projection $p_{y}$ of $K \mathcal{O}_{x} \cap \mathbf{O A}_{y} \cap \mathbf{A}(D) \subset \mathbf{O A}_{y}$ is $p_{y, x}\left(t_{D}^{-1}\right) \mathcal{O}_{y, x}$ where $t_{y}, t_{D}$ in the local ring $O_{x}$ at $x$ are local equations of $y, D$ near $x, \mathcal{O}_{y, x}$ is the completion of the local ring $O_{y, x}=p_{y, x}\left(O_{x}\right)$ of $y$ at $x$; the local intersection multiplicity of $y$ and $D$ at $x$ is $\operatorname{dim}_{k(x)}\left(O_{x}:\left(t_{y}, t_{D}\right) O_{x}\right)=$ $\operatorname{dim}_{k(x)}\left(\mathcal{O}_{x}:\left(t_{y}, t_{D}\right) \mathcal{O}_{x}\right)=\operatorname{dim}_{k(x)}\left(O_{y, x}: p_{y, x}\left(t_{D}\right) O_{y, x}\right)$ equal to the local multiplicity of $\left.D\right|_{y}$ at $x$, and $p_{y, x}\left(t_{D}^{-1}\right) \mathcal{O}_{y, x}$ coincides with the $x$-part of $\mathbb{A}_{y}\left(\left.D\right|_{y}\right)$. The kernel of $\mathrm{e}_{2}(D) \longrightarrow \mathrm{e}_{y}\left(\left.D\right|_{y}\right)$ is $\mathrm{e}_{2}\left(D-D_{y}\right)$.

Theorem (ADELIC INTERPRETATION OF the InterseCtion PAIRING).

(1) The pairing [, ]: $\operatorname{Div}(S) \times \operatorname{Div}(S) \rightarrow \mathbb{Z}$ is a bilinear symmetric form.

(2) It is invariant with respect to translation by principal divisors.

(3) If $D$ is a divisor whose support does not contain a proper irreducible curve $y$, then $\left[D, D_{y}\right]$ is equal to $\operatorname{deg}_{\mathbb{F}}\left(\left.D\right|_{y}\right)$.

(4) The pairing [, ] coincides with the intersection pairing (, ), in particular,

$$
(E, D)=\chi_{\mathcal{A}_{S}}(0)-\chi_{\mathcal{A}_{S}}(D)-\chi_{\mathcal{A}_{S}}(E)+\chi_{\mathcal{A}_{S}}(D+E) .
$$

Proof. The second property follows from invariance of $\chi_{\mathcal{A}_{S}}$ with respect to translation by principal divisors.

To prove the third property, apply the previous lemma to $-D$ and the zero divisor. Then we obtain $\left[D_{y}, D\right]=\chi_{\mathcal{A}_{y}}(0)-\chi_{\mathcal{A}_{y}}\left(-\left.D\right|_{y}\right)$. By section $\mathbf{0}$ the latter is equal to virtual dimension $\operatorname{dim}_{\mathbb{F}}\left(\mathbb{A}_{y}(0): \mathbb{A}_{y}\left(-\left.D\right|_{y}\right)\right)$, i.e. to $\operatorname{deg}_{\mathbb{F}}\left(\left.D\right|_{y}\right)=\sum_{x \in y} \operatorname{dim}_{\mathbb{F}}\left(\mathcal{O}_{\left.D\right|_{y}, x}: 0\right)$. 
To prove the first property, we first use a standard argument applied to the adelic intersection pairing. For divisors $E_{1}, E_{2}$, the moving lemma [Li, 9.1.10] allows to find linearly equivalent to them divisors $E_{1}^{\prime}, E_{2}^{\prime}$ such that their support does not contain $y$. Therefore, by the previous paragraph and additivity of the degree, $\left[D_{y}, E_{1}+E_{2}\right]=$ $\left[D_{y}, E_{1}^{\prime}+E_{2}^{\prime}\right]=\left[D_{y}, E_{1}^{\prime}\right]+\left[D_{y}, E_{2}^{\prime}\right]=\left[D_{y}, E_{1}\right]+\left[D_{y}, E_{2}\right]$. It is easy to check that for three divisors $D_{1}, D_{2}, D_{3}$, the difference $\left[D_{1}+D_{2}, D_{3}\right]-\left[D_{1}, D_{3}\right]-\left[D_{2}, D_{3}\right]$ is symmetric in $D_{i}$. Since it is 0 when $D_{3}=D_{y}$, it is zero when $D_{2}=D_{y}$, i.e. $\left[D_{y}, E\right]+[C, E]=$ $\left[D_{y}+C, E\right]$ for all divisors $C, E$. The rest is automatic: then $\left[D_{y}+D_{y^{\prime}}, E\right]+[C, E]=$ $\left[D_{y}, E\right]+\left[D_{y^{\prime}}, E\right]+[C, E]=\left[D_{y}+C, E\right]+\left[D_{y^{\prime}}, E\right]=\left[D_{y}+D_{y^{\prime}}+C, E\right]$ and so on, so for every $D>0, C, E$ we have $[D, E]+[C, E]=[D+C, E]$. Write an arbitrary $D$ as $D_{1}-D_{2}$ with $D_{1}, D_{2}>0$. Then $\left[D_{2}, E\right]+[D, E]=\left[D_{1}, E\right]$ and $[D+C, E]+\left[D_{2}, E\right]=$ $\left[D_{1}+C, E\right]=\left[D_{1}, E\right]+[C, E]$, so $[D, E]+[C, E]=[D+C, E]$.

The properties (1), (3), (2) and the use of the moving lemma uniquely characterize the intersection pairing.

Thus, we can compute the intersection number of two divisors entirely in terms of adelic objects associated to the adelic complexes for the divisors.

\section{REMARKS.}

1. The previous presentation was chosen to use as little nontrivial results from algebraic geometry as possible. If one decides to use Bertini's theorem which implies that every divisor is represented as $D_{y_{1}}-D_{y_{2}}$ modulo a principal divisor, where $y_{i}$ are smooth irreducible curves, then $[D, E]=\left[D_{y_{1}}, E\right]-\left[D_{y_{2}}, E\right]$ and each of the terms on the right hand side is linear in $E$, hence so is $[D, E]$. As remarked by one of the referees, one then needs to be careful about the use of Bertini's theorem in the case of finite field $\mathbb{F}$. One can notice that for a finite extension $\mathbb{F}^{\prime}$ of $\mathbb{F}$ there is a straightforward relation between the adelic complexes $\mathcal{A}_{S}$ and $\mathcal{A}_{S^{\prime}}$ where $S^{\prime}=S \times_{\mathbb{F}} \mathbb{F}^{\prime}$. Denote $j: S^{\prime} \longrightarrow S$. For divisors $D, E$ on $S$ we get $\chi_{\mathcal{A}_{S^{\prime}}}\left(j^{*} D\right)=\left|\mathbb{F}^{\prime}: \mathbb{F}\right| \chi_{\mathcal{A}_{S}}(D),\left[j^{*} D, j^{*} E\right]_{S^{\prime}}=\left|\mathbb{F}^{\prime}: \mathbb{F}\right|[D, E]_{S}$. For a given $D$ find a finite extension $\mathbb{F}^{\prime}$ of $\mathbb{F}$ such that $j^{*}(D)$ is the difference of the divisors of smooth proper irreducible curves on $S^{\prime}$ modulo principal divisors and deduce the linearity of $[D, E]_{S}$ with respect to $E$.

2. Another $K$-theoretic adelic interpretation of the intersection index is given in [P2,§2]. That approach seems to be more difficult to apply to derive a short proof of the following corollary.

Adelic Riemann-Roch theorem for 1-Cycles on $S$.

$$
(D, \mathfrak{C}-D)=\chi_{\mathcal{A}_{S}}(0)-\chi_{\mathcal{A}_{S}}(D)-\chi_{\mathcal{A}_{S}}(\mathfrak{C}-D)+\chi_{\mathcal{A}_{S}}(\mathfrak{C})=2\left(\chi_{\mathcal{A}_{S}}(0)-\chi_{\mathcal{A}_{S}}(D)\right) .
$$

Finally, similar to dimension one and the argument at the end of section 0 , we use the isomorphisms $H^{i}\left(\mathcal{A}_{S}(D)\right) \simeq H^{i}\left(S, \mathcal{O}_{S}(D)\right.$ ) established in [P1, Thm1] (for rational adeles), [B1], [H] for full geometric adeles, to get another corollary

Riemann-Roch theOREm For 1-CyCles on $S$.

$$
(D, \mathfrak{C}-D)=2\left(\chi\left(S, \mathcal{O}_{S}(0)\right)-\chi\left(S, \mathcal{O}_{S}(D)\right)\right)
$$




\section{SeVERAL MORE REMARKS AND PROBlems.}

1. Study functorial properties of the adelic complex with respect to morphisms of surfaces and their applications. Extend the argument in this paper to the case of a quasi-coherent sheaf $\mathcal{F}$ on $S$ and the associated adelic complex $\mathcal{A}_{S}(\mathcal{F})$ defined in [B1]. Find an adelic proof of the Noether formula and the Hodge index theorem.

2. Theorems for 1-cocycles and 0 -cycles. The adelic proofs of the Riemann-Roch theorems for 1-cocyles on curves and surfaces over fields do not actually require more than adelic duality. In contrast, the study of 0-cycles on surfaces is more in need of such a harmonic analysis theory. See $[F 4, \S 3.6]$ for the theory of analytic adeles $\mathbb{A}$, measure, integration and harmonic analysis on associated objects, and a two-dimensional theta-formula on elliptic surfaces. The latter is closely related to the Riemann-Roch theorem for zero cycles on surfaces $[\mathrm{F} 4, \S 56, \mathrm{Rk} 3]$.

3. On arithmetic extension. Let $S \rightarrow$ Spec $O_{\mathrm{k}}$ be a regular proper scheme of relative dimension one, $\mathrm{k}$ a number field. The objects $\mathbf{A}, \mathbf{B}, \mathbf{C}$ were defined in $[\mathrm{F} 4, \S 28]$ and their properties were listed. In particular, $\mathbf{A}$ are self-dual with respect to a homomorphism $\mathbf{A} \longrightarrow \mathbb{C}_{1}^{\times}$. See [M2] for details of the proof of two additive reciprocity laws $\mathbf{B}^{\perp} \supset \mathbf{B}$, $\mathbf{C}^{\perp} \supset \mathbf{C}$. It is a fundamental problem to find an analogue $\mathcal{A}_{S}$ of the adelic complex in the arithmetic case and an extension of the adelic Euler characteristic, which gives in particular an adelic description of the Arakelov intersection index and another proof of the Faltings Euler characteristic theorem $[\mathrm{Fa}]$.

4. On Galois equivariant theorems. Galois equivariant adelic Riemann-Roch theorem for certain noncommutative finite group bundles on arithmetic surfaces flat over $\mathbb{Z}$, whose proof uses $K$-delic structures, are studied in the recent [CPT]. It is an interesting problem to develop an approach to equivariant Riemann-Roch theorems using the additive adelic structures approach in this paper.

\section{Bibliography}

[A] E. Artin, Algebraic numbers and algebraic functions, Nelson 1968.

[B1] A. Beilinson, Residues and adeles, Funct. Anal. and Appl. 14(1980), 34-35.

[B2] A. Beilinson, How to glue perverse sheaves, in K-theory, Arithmetic and Geometry (Moscow 1984), LNM 1289, Springer, 1987, 42-51.

[Bo] N. Bourbaki, Commutative algebra, Springer 1989.

[C1] A. Cámara, Topology on rational points over higher local fields, arXiv:1106.0191

[C2] A. Cámara, Functional analysis on two-dimensional local fields, arXiv:1210.2995

[CPT] T. Chinburg, G. Pappas, M.J. Taylor, Higher adeles and non-abelian Riemann-Roch, arXiv: 1204.4520

[Fa] G. Faltings, Calculus on arithmetic surfaces, Ann. Math. 119(1984) 387-424.

[F1] I. Fesenko, Sequential topologies and quotients of Milnor K-groups of higher local fields, Algebra i Analiz, 13 (2001), issue 3, 198-221; English transl. in St. Petersburg Math. J. 13 (2002), 485-501. 
[F2] I. Fesenko, Analysis on arithmetic schemes. I, Docum. Math. Extra Volume Kato (2003) 261-284.

[F3] I. Fesenko, Adelic approach to the zeta function of arithmetic schemes in dimension two, Moscow Math. J. 8(2008), 273-317.

[F4] I. Fesenko, Analysis on arithmetic schemes. II, J. K-theory 5(2010), 437-557.

[F5] I. Fesenko, 2d zeta integral, geometry of $2 \mathrm{~d}$ adeles and the BSD conjecture for elliptic curves over global fields (Analysis on arithmetic schemes. III), work in progress.

[FK] I. Fesenko, M. Kurihara (eds.), Introduction to higher local fields, Geometry and Topology Monographs, vol. 3, 2000, available from www.maths.nottingham.ac.uk/personal/ibf/volume.html

[G] B. Green, On the Riemann-Roch theorem for orders in the ring of valuation vectors of a function field, Manuscr. Math. 60(1988), 259-276.

[H] A. Huber, On the Parshin-Beilinson adeles for schemes, Abh. Math. Sem. Univ. Hamburg 61(1991), 249-273.

[HY1] R. Hübl, A. Yekutieli, Adeles and differential forms, J. Reine Angew. Math. 471(1996), 1-22.

[HY2] R. Hübl, A. Yekutieli, Adelic Chern forms and applications, Amer. J. Math. 121(1999), 797-839.

[Iw1] K. Iwasawa, Letter to J. Dieudonne (April 1952), reproduced in Adv. Stud. Pure Math., 21, Zeta functions in geometry, 1992, 445-450.

[Iw2] K. Iwasawa, On the rings of valuation vectors, Ann. Math. 57(1953), 331-356.

[Iw3] K. Iwasawa, Algebraic functions, Transl. Math. Monogr., vol. 118, AMS, 1993.

[Ko] G. Köthe, Topological vector spaces. I, Springer 1969.

[Kp] M. Kapranov, Semiinfinite symmetric powers, math.QA/0107089.

[KS] K. Kato, Sh. Saito, Two-dimensional class field theory, Adv. Stud. Pure Math., vol. 2, 1983, 103-152.

[Kt] K. Kato, Existence theorem for higher local fields, in Invitation to higher local fields, I. Fesenko and M. Kurihara, (eds.), Geometry and Topology Monographs Vol 3, 2000, 165-195.

[Le] S. Lefschetz, Algebraic topology, Amer. Math. Soc. 1942.

[Li] Q. Liu, Algebraic geometry and arithmetic curves, Oxford Univ. Press 2002.

[M1] M. Morrow, An explicit approach to residues on and dualizing sheaves of arithmetic surfaces, New York J. Math. 16(2010), 575-627.

[M2] M. Morrow, Grothendieck's trace map for arithmetic surfaces via residues and higher adeles, J. Algebra and Number Th. 6-7 (2012), 1503-1536.

[M3] M. Morrow, An introduction to higher dimensional local fields and adeles, arXiv:1204.0586

[OP1] D.V. Osipov, A.N. Parshin, Harmonic analysis on local fields and adelic spaces. I. (Russian) Izv. Ross. Akad. Nauk Ser. Mat. 72 (2008), no. 5, 77-140; Engl. transl. in Izv. Math. 72 (2008), no. 5, 915-976; II. (Russian) Izv. Ross. Akad. Nauk Ser. Mat. 75 (2011), no. 4, 91-164; Engl. transl. in Izv. Math.75 (2011), no. 4, 749-814.

[OP2] D.V. Osipov, A.N. Parshin, Harmonic analysis and Riemann-Roch theorem, arXiv: 1107.0408

[P1] A.N. Parshin, On the arithmetic of two dimensional schemes. I, Distributions and residues, Math. USSR Izv. 10(1976), 695-729.

[P2] A.N. Parshin, Chern classes, adeles and L-functions, J. Reine Angew. Math. 341 (1983), 174-192.

[Pr] L. Previdi, Locally compact objects in exact categories, Intern. J. Math. 22(2011) 1787-1821. 
[S] J.-P. Serre, Groupes algébriques et corps de classes, 2nd ed., Hermann, Paris, 1975.

[T] J. Tate, Fourier analysis in number fields and Hecke's zeta function, $\mathrm{PhD}$ thesis, Princeton Univ., 1950; reproduced in Algebraic number theory, eds. J. Cassels and A. Fröhlich, Academic Press, 1967.

[W] A. Weil, Basic number theory, Springer, 1967.

[Y] A.Yekutieli, An explicit construction of the Grothendieck residue complex, Astérisque 208, 1992.

[Z] I. Zhukov, Higher dimensional local fields, in I. Fesenko and M. Kurihara (eds.) Invitation to higher local fields, Geometry and Topology Monographs, vol. 3, 2000, 5-18. 